\title{
The End of Symbolic Exclusion? The Rise of "Categorical Tolerance" in the Musical Tastes of Americans: 1993-2012
}

\author{
Omar Lizardo, Sara Skiles
}

University of Notre Dame

Abstract: In this article, we aim to contribute to recent work in the sociology of taste on the role of cultural dislikes as resources for symbolic exclusion and identity construction. We merge a new data set that replicates musical taste (patterns of likes and dislikes) items from the 1993 GSS with a new data source, resulting in the first repeated cross-section on patterns of likes and dislikes in the U.S. population. Our key finding is that there has been a dramatic shift in the way that people from the United States use cultural dislikes for purposes of symbolic exclusion: namely, the rise of a significant segment of the population that refuses to use culture for this purpose. To shed further light on this pattern, we deploy a statistical model that allows us to distinguish respondents who could have expressed dislikes but did not from those who were predisposed to not dislike any cultural form from the very beginning. The results show that the main drivers of the shift towards "refusing to dislike" are very likely cohort replacement and the increasing "browning" of the American population.

Keywords: symbolic exclusion; musical taste; cultural dislikes; categorical tolerance

Citation: Lizardo, Omar, and Sara Skiles. 2016. "The End of Symbolic Exclusion? The Rise of "Categorical Tolerance" in the Musical Tastes of Americans: 1993 - 2012." Sociological Science 3: 85-108.

Received: July 26, 2015

Accepted: August 3, 2015

Published: February 11, 2016

Editor(s): Jesper Sørensen, Gabriel Rossman

DOI: $10.15195 / \mathrm{v} 3.25$

Copyright: (C) 2016 The Author(s). This open-access article has been published under a Creative Commons Attribution License, which allows unrestricted use, distribution and reproduction, in any form, as long as the original author and source have been credited. (0)(1) key insight of recent research in the sociology of taste is that patterns of cultural A "likes" and "dislikes" provide empirical purchase on how cultural distancing and symbolic boundary-drawing processes enter into identity-construction projects at a collective level (Bryson 1996; Bourdieu 1984; Goldberg 2011; Tampubolon 2008; Lamont and Molnar 2004). The basic idea is that rejection of certain cultural forms-what Bryson (1996:886) has referred to as "symbolic exclusion"-is a proxy for rejecting the persons that are perceived to be the audiences of those forms (social exclusion). Both symbolic and social exclusion are, in their turn, intimately tied to identity-construction projects (Bryson 1997). In this respect, judgments of taste (both positive and negative) can serve simultaneously as markers of where the boundary separating "us" from "them" resides, signals to both in-group and out-group members about the scope and extent of those boundaries, and claims of authenticity and membership directed at in-group others (Bryson 1997; Carter 2006; Lamont and Lareau 1988).

\section{Symbolic Exclusion and Cultural Taste Patterns: Two Themes}

\section{The Classical Model: Taste as Vertical Distinction}

The literature on how cultural taste plays into symbolic exclusion has been dominated by two overarching themes. First is the role of cultural tastes (both acceptances and rejections) in signaling class membership and class differences (Bourdieu 1984; 
Bryson 1996, 1997; Peterson and Simkus 1992). The basic idea here is that by rejecting so-called low-status cultures, high-status persons both constitute and reaffirm the basis of their hierarchical differences in relation to the groups associated with the cultures so rejected (Bourdieu 1984). The main empirical prediction is that symbolic exclusion should flow from the top to the bottom of the status ladder, with the privileged drawing boundaries in relation to the culture preferred by nonelites.

The main empirical puzzle that confronted this research was the discovery of the phenomenon of patterned tolerance among high-status individuals (Bryson 1996), with "tolerance," a term adapted by Bryson (1996) from Stouffer's (1955) classic research on political and social attitudes, being conceptualized as the reticence (or refusal) to express a large number of dislikes. This is a puzzle from the perspective of the classical model, since the basic prediction is that high-status individuals required the active rejection of low-status tastes in order to actively reproduce and legitimize their access to valuable resources and positions of authority (Bourdieu and Passeron 1977).

Contrary to this prediction, Bryson (1996) found that rather than actively rejecting a wide swath of nonelite culture, high-status persons are less likely to actively express dislike for (certain forms of) nonelite culture. This finding has been replicated multiple times both in the United States and in other empirical contexts (e.g., van Eijck 2001; Warde, Wright, and Gayo-Cal 2008; Tampubolon 2008; Lizardo 2005; Goldberg 2011; Savage and Gayo 2011). This line of research concludes that for high-status elites, patterned tolerance (and thus a relative reticence to express dislikes for certain cultural styles) serves primarily as an identity signal of "openness to diversity" coupled with a rejection of imagined others who are perceived as intolerant and narrow minded (Ollivier 2008; Prieur and Savage 2011; Holt 1997). Thus relatively tolerant tastes are increasingly used (primarily by high-status persons) as resources to construct a cosmopolitan, "modern" self, capable of integrating and enjoying a wide range of cultural styles and experiences. This is what Bryson (1996) has referred to as "multicultural capital" and Ollivier (2004) has dubbed "conspicuous openness to diversity."

This emerging institutionalized reticence to engage in (overt) symbolic exclusion maps to structural inducements generated by the relational position of high-status elites, insofar as openness to a wide variety of styles is correlated with the capacity to navigate within and between cross-cutting, complex circles (DiMaggio 1987, 1996; Ollivier 2008; Bryson 1996; Lizardo 2006; Collins 1975). This is related to high-status individuals' capacity to connect to emergent institutional discourses located at the world-culture level (e.g., Boli and Lechner 2005), which denigrates symbolic exclusion as tied to such collectively recognized evils as racism, prejudice, and ethnocentrism (Ollivier 2008; Prieur and Savage 2011; Lizardo 2008). Increasingly entrenched institutional rules define a capable (post)modern self as one who refuses to exclude (symbolically and, by implication, socially). The modern individual should thus give all cultural styles a potential chance to enter into identity-constitution projects at the personal level, decoupling individual identity formation from exclusionary collective identity-constitution projects (Lizardo 2005; Frank and Meyer 2002). 


\section{Taste as a Collective Resource for Identity Construction}

This leads us to the second theme that has preoccupied the literature on symbolic exclusion: namely, the role of cultural tastes in the construction of collective identity and group membership. In particular, there is ample evidence to suggest that certain groups are structurally induced to use the rejection of certain styles to signal authentic membership in their respective in-groups (Carter 2006; Mahon 2000; Bryson 1997). Bryson (1997) used the identity-construction argument to explain why U.S. people with low education are more likely to engage in symbolic exclusion than their highly educated counterparts. She found that, among low-education individuals, social categories salient in group formation (e.g., gender, race, religion, ethnicity) better predict symbolic exclusion than they do among highly educated persons. This suggests that among persons who belong to low-status groups, symbolic exclusion is more likely to play a role in identity-constitution processes via social differentiation from horizontally proximate groups defined by race, gender, religion, and other axes of categorical differentiation (Bryson 1997).

\section{Contributions of the Present Work}

In this article, we aim to contribute to these two strands in the sociology of taste, using a new dataset that replicates musical taste (patterns of likes and dislikes) items from the 1993 General Social Survey (GSS). We merge this data with the original GSS culture module, providing us with the first repeated cross-section-covering about two decades - on patterns of likes and dislikes of the U.S. population. Our key finding is that there has been a dramatic shift in the way that people in the United States use cultural dislikes for symbolic exclusion purposes: namely, the growth in the significant segment of the population that refuses to use culture for this purpose. We show that the finding is unlikely due to a method effect (e.g., different data collection methods in our survey versus the GSS), since the shape of the distribution of cultural likes and the truncated distribution of dislikes has remained essentially unchanged.

We use a statistical model that allows us to distinguish respondents who could have expressed dislikes but did not from those who were predisposed to not dislike any cultural forms from the very beginning. This allows us to assess the hypothesis that the bulk of the shift can be explained by increases in the latter type of respondent. The results show that indeed the proportion of respondents who categorically refuse to engage in symbolic exclusion of any form has about tripled in the last two decades, and that the main driver of the change is very likely cohort replacement and the increasing "browning" of the U.S. population. In addition, we show that symbolic exclusion has begun to decline as a low-status location marker, with those with low education in the United States no longer being more likely to express musical dislikes than their highly educated counterparts. In all, the results point to an increasing destructuration of symbolic exclusion as both a class-based symbolic boundary-drawing index (as suggested by previous research) and as a mechanism for collective identity-construction among low-status groups (with the possible exception of race). 


\section{Organization of the Article}

The rest of the article is organized as follows: in the next section we describe our survey and data collection methods, show how we joined these data with the original GSS data, and describe the key variables to be used in the analysis. Following that, we present the main set of descriptive results and present our multivariate analysis of patterns of change in symbolic exclusion. We close by outlining the substantive and theoretical implications of our results for future research in the sociology of taste.

\section{Data and Predictors}

Half of the data for this study come from the 1993 "culture module" of the General Social Survey (hereafter GSS 1993; Davis and Smith 2007). The GSS 1993 culture module $(\mathrm{N}=1,606)$ included items assessing respondents' likes and dislikes (as well as a "mixed feelings" middle category) for 18 musical style categories: big band/swing; bluegrass; rhythm and blues (R\&B); Broadway musicals/show tunes; classical/symphony and chamber; contemporary pop/rock; country and Western; folk; gospel; heavy metal; jazz; Latin/mariachi/salsa; mood/easy listening; new age/space; opera; oldies rock; rap; and reggae. This is now a "canonical" data set providing the empirical basis for a variety of analyses (and re-analyses) in the sociology of taste (e.g., Bryson 1996, 1997; Tampubolon 2008). In the summer of 2012 we fielded a survey that was (partially) designed to replicate the 1993 GSS effort. The data were collected by Survey Sample International (hereafter SSI 2012), a private firm that specializes in sampling, data collection, and analysis. SSI managed recruitment and participation-invitation tasks to generate a panel of adults from which our working sample was drawn. Survey respondents were selected from the panel for participation based on age, gender, race, education, and geographic region in order to approximate a sample representative of the U.S. population $(\mathrm{N}=$ 2276). ${ }^{1}$

\section{Outcome variable}

Our key outcome variable is, following Bryson (1996), an additive index of the number of musical dislikes expressed by the person. Theoretically this is a (linear) index of symbolic exclusion, with higher values indicating a greater propensity to engage in this practice. Of particular interest is the "zero" category, indicating a propensity to "refuse to dislike." We treat this outcome as a count variable and use the appropriate statistical modelling strategy for such outcomes, as we elaborate below.

\section{Predictor Variables}

We use a set of basic socio-demographic as predictors:

- Survey Year, a binary variable that equals 1 for 2012 and 0 for 1993. 
- Cohort, in four categories: (1) born before 1936; (2) born between 1936 and 1954; (3) born between 1955 and 1968; and (4) born after 1968. In multivariate analysis the omitted predictor is that coding for persons born between 1955 and 1968.

- Ethnoracial status: Two binary predictors, one coding for "white" versus "nonwhite" status and another coding for "black" versus "nonblack" status. The implied "omitted category" is persons who identified their ethnoracial status as "other." 2,3

- Education: Binary predictors coding for: (1) having completed a high school degree; (2) having completed a bachelor's degree; and (3) having completed some form of postgraduate education (e.g., MA, $\mathrm{PhD}$, professional degree). In multivariate analyses, the omitted category is persons with no degree.

- Gender: A binary predictor that equals 1 for women and 0 for men.

\section{Results}

\section{The Refusal to Dislike}

We begin by describing the distribution of the number of musical dislikes reported by GSS respondents in 1993 and SSI respondents in 2012. The results are shown in Figure 1. The key shift between the two surveys is obvious: the shape of the distributions is very similar except for the number of persons who report not disliking any genre. In the 1993 GSS data this is little more than five percent of respondents, but the corresponding figure in the 2012 SSI survey is about 16 percent, which is about three times as large. This is a substantively significant increase in this category of respondents in just two decades.

We can formally test the hypothesis that the two distributions are similar except for the "inflated" number of zeros in 2012 by performing a statistical test of distributional equality on both the full and the truncated (excluding the zero category) distributions across surveys. ${ }^{4}$ When comparing the full distribution across surveys, we can safely reject the null hypothesis that the two distributions come from comparable populations $(|z|=7.06 ; p<0.01)$. However, when comparing the truncated distributions, the null hypothesis of no difference cannot be rejected at conventional levels of statistical significance $(|z|=1.68 ; p=0.09)$, suggesting that except for the zero category the distributions of dislikes do come from comparable populations. ${ }^{5}$ This indicates that the primary change across two decades in symbolic exclusion behavior is not in the makeup of the population that expresses any number of dislikes, but in the swelling of the proportion of the population that refuses to engage in symbolic exclusion. Building on Bryson's linkage between likes and dislikes and the notion of tolerance from the political opinion literature, we will refer to these respondents as categorical tolerants and to the general phenomenon of refusing to dislike any genre as categorical tolerance in what follows. 


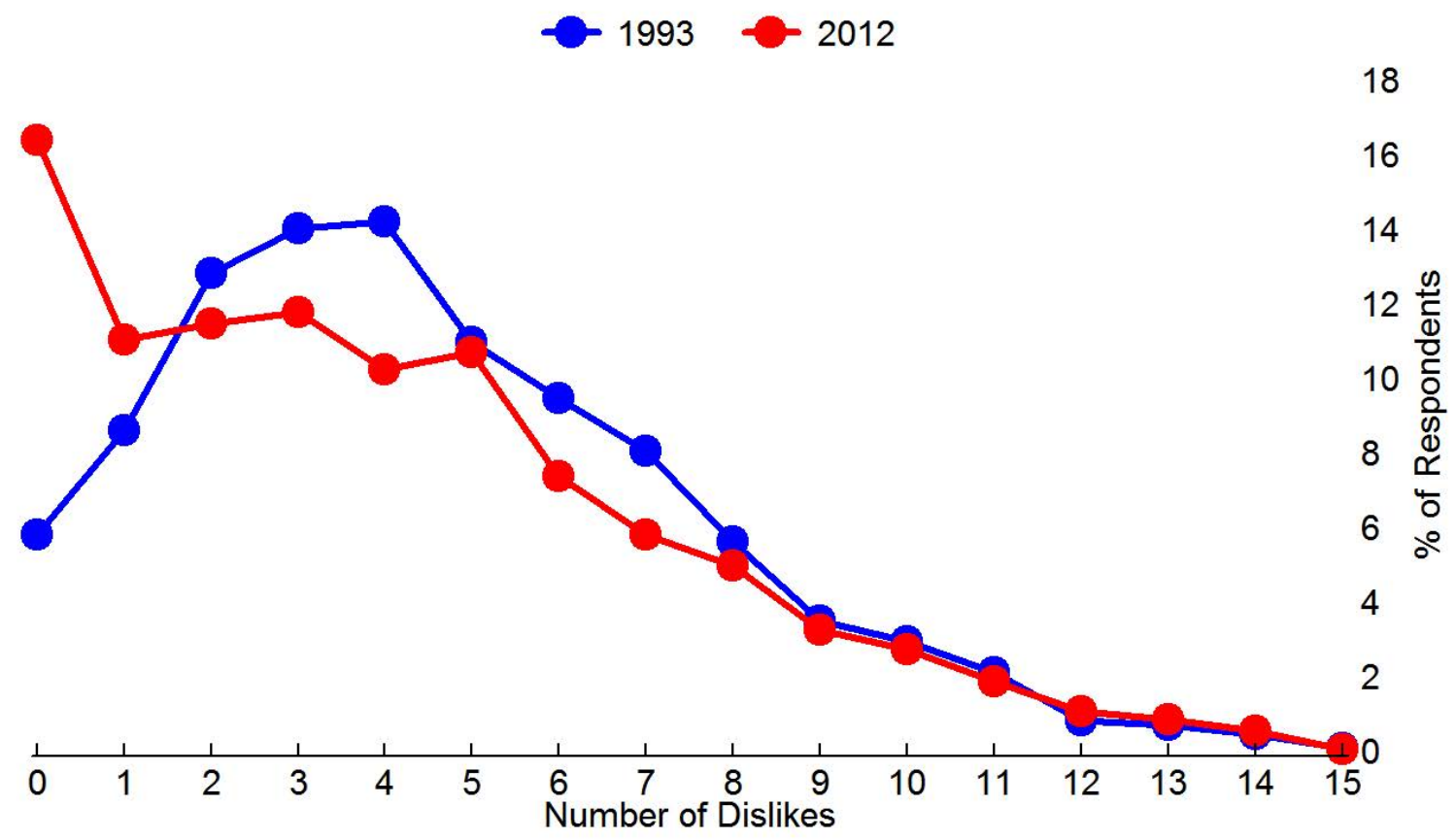

Figure 1: Univariate distribution of counts of the number of dislikes for GSS 1993 (blue) and SSI 2012 (red)

\section{The Rise of Categorical Tolerance: A Method Effect?}

Because such large-scale shifts are rare in social science (see e.g., McPherson, SmithLovin, and Brashears 2006 and Hout and Fischer 2002 for discussion of social changes of similar magnitude in a different empirical context), it is reasonable to treat this finding with skepticism. In particular, the most likely culprit for those who might think that the finding is artifactual is the SSI 2012 survey, which clearly follows a different sampling design and a different administration method than the GSS 1993.

There are three pieces of evidence that mitigate against the hypothesis that the 2012 finding is artifactual. First, if the rise in the proportion of persons who report no dislikes had to do with some peculiarity of the 2012 SSI sample, then we should find the distribution of likes to be equally distorted (e.g., by showing a disproportionate number of persons who like everything). However, as Figure 2 shows, though the SSI and GSS distributions of likes across surveys are not identical $(|z|=3.56 ; p<0.01)$, they are remarkably similar in shape and exhibit no swelling in any one category.

Second, as noted above, the change in the distribution of dislikes between the two samples appears to be confined to those who report no dislikes. Other than that, SSI estimates of persons who report one, two, three, or more dislikes are relatively close to 1993 GSS estimates, and among persons reporting a large number of dislikes they are almost identical. If the rise in the number of persons who refuse to dislike any genre was due to an overall incapacity of the 2012 SSI survey to 


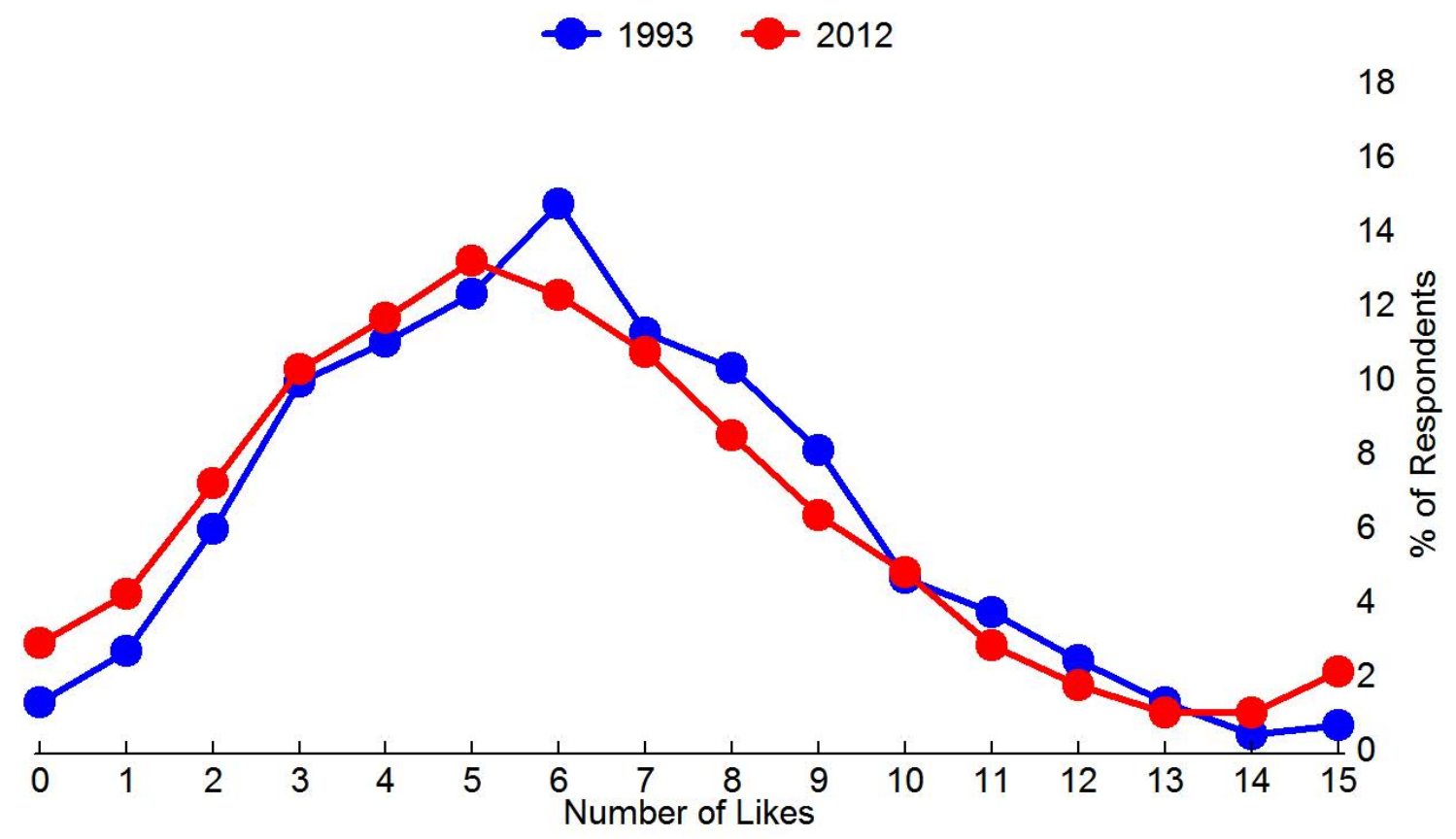

Figure 2: Univariate distribution of counts of the number of likes for GSS 1993 (blue) and SSI 2012 (red)

generate accurate estimates, why should the inaccuracy be confined to this one category of respondents?

Finally, as we will see in Table 1, the SSI survey does a pretty good job of recovering estimates of the average number of dislikes across socio-demographic categories (e.g., average number of dislikes for men and women) with face validity in comparison to the 1993 GSS estimates. If there were something peculiar about the 2012 SSI sampling design, or if there were a method artifact connected with the mode of administration, we should expect some highly divergent results across surveys. However, the differences are relatively minimal and seldom produce counterintuitive results.

\section{Who are the Categorical Tolerants?}

Are categorical tolerants a particularly distinct segment of the population? Table 1 shows the results of a two-sided $t$-test of the null hypothesis that categorical tolerants are different from the rest of the sample along four socio-demographic markers (gender, race, education, and cohort group) for 1993 GSS and 2012 SSI respondents. Four things deserve notice.

First, men and women are equally represented among non-dislikers. In both 1993 and 2012 the proportion of women in each group mirrors that of the sample as a whole. That is, women make up 57 percent of the 1993 sample and 58 percent of the non-dislikers in the same year, and 52 percent of both the sample and the proportion of non-dislikers in 2012. Second, African Americans are overrepresented among non-dislikers on both survey waves by a factor of about two to one (one is 
Table 1: Two sided $t$-test for differences in the probability of expressing no dislikes for binary predictors on gender, race, education, and cohort

\begin{tabular}{lcccccc}
\hline & \multicolumn{2}{c}{1993} & & \multicolumn{2}{c}{2012} \\
& Dislikes $>0$ & Dislikes $=0$ & $p$ & Dislikes $>0$ & Dislikes $=0$ & $p$ \\
\hline Women & 0.57 & 0.58 & 0.89 & 0.52 & 0.52 & 0.82 \\
Whites & 0.85 & 0.69 & 0.00 & 0.67 & 0.51 & 0.00 \\
Blacks & 0.11 & 0.2 & 0.00 & 0.11 & 0.19 & 0.00 \\
H.S. Degree* & 0.59 & 0.53 & 0.26 & 0.55 & 0.6 & 0.04 \\
Bachelor Degree & 0.16 & 0.16 & 0.92 & 0.29 & 0.21 & 0.00 \\
Post-Grad & 0.07 & 0.08 & 0.95 & 0.13 & 0.13 & 0.93 \\
Born before 1936 & 0.24 & 0.29 & 0.23 & 0.07 & 0.04 & 0.03 \\
Born bt 1936-1954 & 0.34 & 0.26 & 0.10 & 0.18 & 0.11 & 0.00 \\
Born bt 1955-1968 & 0.34 & 0.32 & 0.71 & 0.28 & 0.28 & 0.87 \\
Born after 1968 & 0.08 & 0.13 & 0.12 & 0.46 & 0.57 & 0.00 \\
\hline
\end{tabular}

* Includes respondents with a 2 year college degree.

twice as likely to encounter a black respondent in the non-disliker group than in the population as a whole; $\mathrm{p}<0.00$ ). In fact, the 2012 SSI estimate of the proportion of black respondents who report no dislikes is essentially the same as that of the GSS 1993 (about 20 percent). In the very same way, whites are underrepresented among persons who refuse to dislike by comparable odds ratios in both surveys ( 0.78 and 0.75 respectively), suggesting that the overrepresentation of blacks and the underrepresentation of whites among those who refuse to engage in symbolic exclusion has been a relatively constant feature of this respondent group even as their size has grown over the last two decades. Third, the 1993 GSS data show equal representation by education level among non-dislikers and the rest of the sample, but the 2012 SSI data show an underrepresentation of college-educated respondents (21 percent versus 29 percent; $p<0.01$ ), and an overrepresentation of high schooleducated respondents (60 percent versus 55 percent; $p<0.05$ ). ${ }^{6}$ Finally, and most importantly, though there is no relationship between the likelihood of falling into the non-disliker group and birth year in 1993, by 2012 strong cohort effects have emerged, with non-dislikers being underrepresented among the (surviving) members of the pre-WWII generation $(p<0.01)$ and being overrepresented among members of the post-baby boom cohort.

In all, we can conclude that the group of respondents more likely to refuse to express dislikes has some time-constant features (it is less white than the general population) and one key temporally dynamic feature: it is increasingly likely to be composed of individuals who belong to recently born cohorts. This suggests that a cohort-succession/generational-replacement process coupled with a decline in the proportion of the white population may be the leading dynamic contributing to the dramatic rise of persons who refuse to use cultural taste for symbolic exclusion purposes in the general population. 
Table 2: Two-sided $t$-tests for differences in mean number of dislikes for persons who expressed at least one dislike for binary predictors on gender, race, education, and cohort

\begin{tabular}{lcccccc}
\hline & \multicolumn{3}{c}{1993} & \multicolumn{3}{c}{2012} \\
& No & Yes & $p$ & No & Yes & $p$ \\
\hline Women & 5.02 & 4.69 & 0.03 & 5.01 & 4.5 & 0.00 \\
Whites & 4.93 & 4.82 & 0.57 & 4.45 & 4.88 & 0.00 \\
Blacks & 4.82 & 4.91 & 0.73 & 4.75 & 4.67 & 0.73 \\
H.S. Degree* & 4.96 & 4.74 & 0.14 & 4.8 & 4.7 & 0.45 \\
Bachelor Degree & 4.97 & 4.11 & 0.00 & 4.76 & 4.71 & 0.77 \\
Post-Grad & 4.9 & 3.97 & 0.00 & 4.73 & 4.82 & 0.65 \\
Born before 1936 & 4.67 & 5.37 & 0.00 & 4.75 & 4.57 & 0.49 \\
Born bt 1936-1954 & 4.92 & 4.65 & 0.08 & 4.82 & 4.38 & 0.01 \\
Born bt 1955-1968 & 5.00 & 4.51 & 0.00 & 4.84 & 4.48 & 0.02 \\
Born after 1968 & 4.78 & 5.34 & 0.03 & 4.46 & 5.07 & 0.00 \\
\hline
\end{tabular}

* Includes respondents with a 2 year college degree.

\section{Changes in Average Number of Dislikes}

While the most dramatic social change in the last two decades is the increase in categorical tolerance, we are also likely to observe some important changes in the socio-demographic correlates of persons who engage in some form symbolic exclusion. To examine this question, Table 2 shows the average number of dislikes among the same socio-demographic groups examined in Table 1, restricted to respondents who expressed at least one dislike in either survey.

This table highlights several notable findings. First, women are less likely to engage in symbolic exclusion than are men (for instance, the mean number of dislikes for women in 1993 was 4.69 compared to 5.02 for men; $p<0.03$ ), a finding that was first noted by Bryson (1996). The 1993 GSS and 2012 SSI survey estimates of the gender difference are fairly close, suggesting that this gender dynamic has remained constant over time. Racial differences are another matter. While whites were equally likely as nonwhites to engage in symbolic exclusion in the 1993 data, by 2012 a white/nonwhite gap is evident. This gap seems to be driven by increasing tolerance levels among nonwhites (whites have about similar levels of tolerance across surveys: $|\Delta|=0.06 ; p=0.54$ ). The difference in the average number of dislikes for black and nonblack respondents is statistically null in both surveys, although black respondents seem to have become significantly less likely to engage in symbolic exclusion over time $\left(\mu^{1993}=4.91\right.$ versus $\left.\mu^{2012}=4.67\right)$. This has led to a reversal (in 2012) of the white/black ranking on this dimension.

The most important change detectable in the analysis, however, has to do with education. In the 1993 survey, college-educated respondents and holders of professional degrees expressed (on average) fewer dislikes than their less educated counterparts-the phenomenon that Bryson (1996) referred to as "patterned tolerance." By 2012 this difference has disappeared, with persons being equally likely to engage in symbolic exclusion regardless of education level. The rank of holders 
of postgraduate and professional degree who express at least one dislike is now more likely to be populated by persons more likely to engage in symbolic exclusion $(|\Delta|=0.85 ; p<0.01)$ than is the rank of those who hold a bachelor's degree $(|\Delta|=0.60 ; p<0.01$ ), with less educated persons (those who hold only a high school degree) evincing no shift in symbolic exclusion behavior across surveys $(|\Delta|=0.05 ; p=0.72)$. This has led to a reversal of the patterned tolerance effect, with the highly educated being more likely to express dislikes in 2012 than the less educated. This suggests that the patterned tolerance phenomenon Bryson (1996) observed, rather than being a permanent feature of the U.S. educated class, may have itself have been a historically specific characteristic of the late twentieth-century United States.

Finally, when it comes to generational dynamics, we can see that the most distinctive shift has been the increasing tolerance of surviving members of the pre-WWII generation. Though those born before 1936 were less tolerant than their more recently born counterparts in $1993(p<0.01)$, they are indistinguishable in 2012 from the rest $(p=0.49)$. This phenomenon is most likely due to differential mortality of the least tolerant members of this group. In both surveys, both the WWII and the baby boom cohorts are more tolerant than others, while post-baby boomers are significantly less tolerant than their older counterparts. ${ }^{7}$ Note that there is no one-to-one correspondence between these cohort trends and the cohort effects connected to the rise of categorical tolerants. Members of older generations are underrepresented in the categorical tolerant group even as their general tolerance has increased over time. The same can be said for members of the post-baby boom cohort, who have not experienced any discernible increase in tolerance (in fact being slightly less tolerant than their baby boomer parents), but have come to form an increasing proportion of those who refuse to dislike any genres. This suggests that these two groups (persons who engage in some form-however minimal-of symbolic exclusion and those who do not) may represent two distinct segments of the U.S. population. In terms of statistical modelling, we require a framework that distinguishes effectively between these two types of respondents, because modelling the number of dislikes with the presumption that the population consists of a single group of persons (all of whom could in principle engage in symbolic exclusion) will produce misleading estimates of the relevant causal dynamics.

\section{Statistical Model}

In what follows, we model the socio-demographic correlates of categorical tolerance and symbolic exclusion by presuming that the rise of categorial tolerance is best understood as implying increasing population heterogeneity with respect to categorical tolerance. This heterogeneity divides those who could engage in symbolic exclusion from categorical tolerants, who refuse to do so under any circumstance (at least in the realm of musical taste). The "excess" number of zeros in the SSI 2012 counts of number of musical styles disliked, rather than being a statistical nuisance to be wished away, in fact represents the most important substantive aspect of social change in the symbolic boundary behavior of the U.S. population in the last two decades, and thus should be explicitly modelled. 
We rely on an analytic strategy that is designed to explicitly model (latent) population heterogeneity with a count outcome. ${ }^{8}$ The basic idea is to use a binary logit to first predict the odds of respondents falling into the "non-symbolic excluders" category, and then use a count regression (e.g., Poisson) model to predict the number of dislikes among persons who are at risk of engaging in some form of symbolic exclusion if they cross the first (binary) "hurdle" (by disliking any genres). The basic presumption in regards to the data-generating process is that these two segments of the population are mutually exclusive. Individuals in the first group of true categorical tolerants would not use cultural dislikes to engage in symbolic exclusion under any circumstances, and would therefore produce a ("structural") zero response no matter what. Those in the second group could dislike any number of genres and thus produce a response ranging from zero to the maximum of 15 .

\section{Multivariate results}

One feature of the modelling strategy proposed here is that the specification of the logit model predicting membership in the categorical tolerant group can be different from that predicting levels of symbolic exclusion. We use the preliminary results shown in Tables 1 and 2 (which give us a clue as to which variables matter in both components) to inform our model specification in terms of which inputs and which predictors should be included in both equations and the order in which they should be introduced. We follow a hierarchical regression approach, introducing variables of interest gradually and comparing model fit for each specification.

Table 3 shows the results. Model 1 presents coefficient estimates from a standard (robust) Poisson regression model of total number of musical styles disliked that includes the main socio-demographic predictors. This model will serve as a reference. In this model, cohort enters as a set of three binary predictors (to account for the nonlinear cohort effects shown in Table 2), and survey year enters as a binary predictor that equals 1 for respondents in the SSI 2012 sample and 0 otherwise. The rest of the predictors are binary indicators for the socio-demographic markers of interest (as shown in Tables 1 and 2).

The baseline model gives results consistent with those from the bivariate analyses displayed in Table 1 in terms of race, gender, education, age cohort, and period effect (measured by survey wave). Of particular interest is the curvilinear nature of the cohort effect, in which we see that the youngest and oldest members of the 2012 sample are engaging in symbolic exclusion, likely in an attempt to reject each other's cultures (Tampubolon 2008; Goldberg 2011).

Model 2 shows the respective coefficient estimates for the hurdle count model accounting for the inflation of the zero category across surveys. The Poisson portion of the model is specified in the same way as in model 1, but the binary portion includes both year of survey and a (linear) term for birth year as its main predictors. The basic hypothesis to be tested is that the rise of categorical tolerants is accounted for by the emergence of heterogeneity in the population between those who refuse to dislike any genres and those who are at risk of disliking all genres but who could incidentally express no dislikes. The formal test of the hypothesis pits the standard Poisson model (which assumes that everybody is at risk of expressing 
Table 3: Coefficient Estimates of robust Zero-Inflated Poisson models predicting the number of dislikes in the pooled 1993 GSS and 2012 SSI surveys. Poisson count model estimates are shown in the top-half and binary logit model estimates in the bottom-half. $(\mathrm{N}=3875)$

\begin{tabular}{|c|c|c|c|c|c|}
\hline & (1) & $(2)$ & (3) & $(4)$ & (5) \\
\hline \multicolumn{6}{|l|}{ Poisson Estimates } \\
\hline Year (2012) & $\begin{array}{r}-0.125^{*} \\
(0.026)\end{array}$ & $\begin{array}{l}-0.04 \\
(0.024)\end{array}$ & $\begin{array}{c}-0.041 \\
(0.024)\end{array}$ & $\begin{array}{l}-0.04 \\
(0.024)\end{array}$ & $\begin{array}{l}-0.1^{*} \\
(0.028)\end{array}$ \\
\hline Born before 1936 & $\begin{array}{c}0.106^{*} \\
(0.035)\end{array}$ & $\begin{array}{c}0.102^{*} \\
(0.033)\end{array}$ & $\begin{array}{r}0.102^{*} \\
(0.033)\end{array}$ & $\begin{array}{c}0.102^{*} \\
(0.033)\end{array}$ & $\begin{array}{r}0.085^{*} \\
(0.033)\end{array}$ \\
\hline Born between 1936 and 1954 & $\begin{array}{c}0.028 \\
(0.031)\end{array}$ & $\begin{array}{c}-0.001 \\
(0.029)\end{array}$ & $\begin{array}{c}-0.001 \\
(0.029)\end{array}$ & $\begin{array}{c}-0.001 \\
(0.029)\end{array}$ & $\begin{array}{c}-0.002 \\
(0.029)\end{array}$ \\
\hline Born after 1968 & $\begin{array}{c}0.119^{*} \\
(0.034)\end{array}$ & $\begin{array}{c}0.162^{*} \\
(0.030)\end{array}$ & $\begin{array}{c}0.161^{*} \\
(0.030)\end{array}$ & $\begin{array}{c}0.161^{*} \\
(0.030)\end{array}$ & $\begin{array}{r}0.155^{*} \\
(0.030)\end{array}$ \\
\hline White & $\begin{array}{c}0.163^{*} \\
(0.032)\end{array}$ & $\begin{array}{r}0.080^{*} \\
(0.029)\end{array}$ & $\begin{array}{r}0.076^{*} \\
(0.029)\end{array}$ & $\begin{array}{l}0.076^{*} \\
(0.029)\end{array}$ & $\begin{array}{r}0.081^{*} \\
(0.029)\end{array}$ \\
\hline Woman & $\begin{array}{r}-0.083^{*} \\
(0.024)\end{array}$ & $\begin{array}{r}-0.098^{*} \\
(0.022)\end{array}$ & $\begin{array}{r}-0.097^{*} \\
(0.022)\end{array}$ & $\begin{array}{r}-0.097^{*} \\
(0.022)\end{array}$ & $\begin{array}{r}-0.096^{*} \\
(0.022)\end{array}$ \\
\hline Bachelor Degree & $\begin{array}{c}-0.047 \\
(0.029)\end{array}$ & $\begin{array}{r}-0.082^{*} \\
(0.026)\end{array}$ & $\begin{array}{r}-0.083^{*} \\
(0.026)\end{array}$ & $\begin{array}{r}-0.086^{*} \\
(0.026)\end{array}$ & $\begin{array}{r}-0.216^{*} \\
(0.042)\end{array}$ \\
\hline Professional/Post-Graduate & $\begin{array}{r}-0.081^{*} \\
(0.041)\end{array}$ & $\begin{array}{r}-0.086^{*} \\
(0.036)\end{array}$ & $\begin{array}{r}-0.087^{*} \\
(0.036)\end{array}$ & $\begin{array}{r}-0.087^{*} \\
(0.036)\end{array}$ & $\begin{array}{r}-0.259^{*} \\
(0.062)\end{array}$ \\
\hline Year $\times$ Bachelor & & & & & $\begin{array}{r}0.201^{*} \\
(0.053)\end{array}$ \\
\hline Year $\times$ Post-Graduate & & & & & $\begin{array}{r}0.261^{*} \\
(0.076)\end{array}$ \\
\hline Constant & $\begin{array}{l}1.390^{*} \\
(0.041)\end{array}$ & $\begin{array}{r}1.532^{*} \\
(0.038)\end{array}$ & $\begin{array}{l}1.536^{*} \\
(0.037)\end{array}$ & $\begin{array}{c}1.536^{*} \\
(0.037)\end{array}$ & $\begin{array}{r}1.565^{*} \\
(0.038)\end{array}$ \\
\hline \multicolumn{6}{|l|}{ Logit Estimates } \\
\hline Year (2012) & & $\begin{array}{l}1.047^{*} \\
(0.145)\end{array}$ & $\begin{array}{l}1.053^{*} \\
(0.144)\end{array}$ & $\begin{array}{c}1.101^{*} \\
(0.147)\end{array}$ & $\begin{array}{r}1.121^{*} \\
(0.148)\end{array}$ \\
\hline Birth Year & & $\begin{array}{r}0.012^{*} \\
(0.064)\end{array}$ & $\begin{array}{r}0.011^{*} \\
(0.065)\end{array}$ & $\begin{array}{c}0.011^{*} \\
(0.065)\end{array}$ & $\begin{array}{r}0.011^{*} \\
(0.065)\end{array}$ \\
\hline Black/African-American & & & $\begin{array}{r}0.648^{*} \\
(0.143)\end{array}$ & $\begin{array}{c}0.629^{*} \\
(0.144)\end{array}$ & $\begin{array}{r}0.633^{*} \\
(0.144)\end{array}$ \\
\hline Bachelor Degree & & & & $\begin{array}{r}-0.345^{*} \\
(0.139)\end{array}$ & $\begin{array}{r}-0.351^{*} \\
(0.139)\end{array}$ \\
\hline Constant & & $\begin{array}{r}-26.272^{*} \\
(0.216)\end{array}$ & $\begin{array}{r}-24.781^{*} \\
(0.215)\end{array}$ & $\begin{array}{r}-24.488^{*} \\
(0.216)\end{array}$ & $\begin{array}{r}-24.217^{*} \\
(0.217)\end{array}$ \\
\hline Model $\chi^{2}$ & 103.7 & 89.22 & 88.54 & 89.14 & 114.64 \\
\hline
\end{tabular}

$* p<0.05$ (two-tailed tests). 
some dislikes) against the mixed binary-count model (which assumes a bifurcation in the population). The result of this test decisively rejects the hypothesis of population homogeneity of model 1 , favoring the (latent population heterogeneity) specification shown in model $2(z=14.17, p<0.001){ }^{9}$

As the lower half of the second column (logit estimates) shows, there is a strong period effect of survey year. Respondents are close to three times $\left(e^{1.05}=2.8\right)$ more likely to fall into the categorical tolerant group in 2012 than they were in 1993. Note also that, after adjusting for socio-demographic heterogeneity, the negative effect of survey wave shown in the count component of the model decreases by about one-third (in relation to the simple Poisson estimate in model 1) and is no longer statistically significant. Thus what could have been mistaken for a period trend in tolerance (when it comes to a decrease in the expected number of genres disliked) is actually the spurious result of the growth in the population that is not at risk of disliking any genres. Once we account for this factor, we find that there is in fact no difference between the two surveys in the average number of genres disliked among who are at risk of engaging in some form of symbolic exclusion.

There are other substantively significant differences between models 1 and 2 . First note that the effect of being white is reduced by about 50 percent. This suggests that about half of the white intolerance effect is actually a result of their relative under-representation in the (growing) group of categorical tolerants. The same can be said for the effect of holding a bachelor's degree in comparison to having a high school degree or being a high school dropout. Though in model 1 college-educated respondents were indistinguishable from others, in model 2 the expected "educated tolerance" effect emerges. This is due to the fact that persons with a bachelor's degree are relatively under-represented among categorical tolerants (as model 4 confirms). Once we account for population heterogeneity, Bryson's educationtolerance gradient can be observed. Finally, note the statistically significant effect of the linear cohort predictor binary part of the model $(t=3.55)$. This indicates that the more recently a respondent was born, the more likely he or she is to belong to the categorical tolerant group. Note that this is in spite of the fact that those born after 1968 are also less tolerant than their baby boomer elders $(t=5.31)$. This is not contradictory, since the latter belong to the population of persons who are at risk of engaging in symbolic exclusion (the model presumes that these two populations are disjoint within levels of any one socio-demographic factor). The substantive message is that the rise of categorical tolerants goes in tandem with the relative survival (and possible revival) of taste-based symbolic exclusion among members of the same generation (Bryson 1996).

Models 3-5 adjust the specification to account for additional socio-demographic effects on both symbolic exclusion (number of dislikes) and odds of falling into the categorically tolerant group. Models 3 and 4 reveal the same race and education effects described in the bivariate analyses above. In model 5 we account for the decline of the educated tolerance effect evident in the bivariate analysis by including an interaction between the education predictors and survey wave for both persons with a bachelor's degree and persons with a professional or postgraduate degree. As expected, we find that the moderating effect of survey wave for highly educated persons is positive, indicating that in the more recent wave there is no 


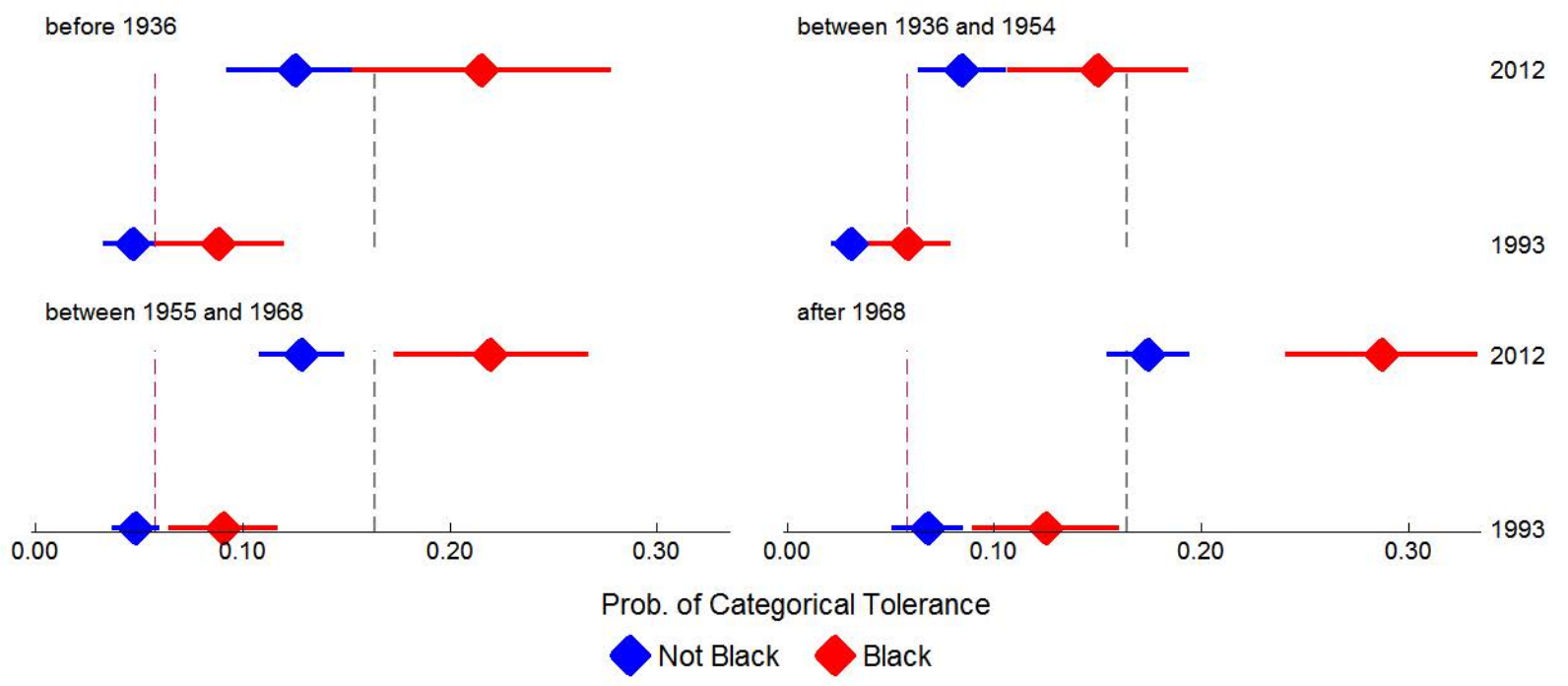

Figure 3: Predicted probability of being a categorical tolerant from binary component of the preferred zeroinflated Poisson specification by cohort group and ethnoracial status. Dashed vertical lines indicate sample estimates from proportion of the population with zero dislikes in 1993 (red dashes) and 2012 (gray dashes)

education-based gradient on tolerance (e.g., $-0.259+0.261=0.02$ for persons with a postgraduate degree). This supports our initial impression that highly educated persons became less tolerant from 1993 to 2012. Note however that the negative period effect for survey wave is once again statistically significant in the multiplicative specification $(t=-3.50)$. There is nothing strange about this. Due to the presence of a multiplicative predictor involving this covariate, this is now a group-specific period effect applying only to persons without a college or professional degree. The substantive message is that during the same period in which their more highly educated counterparts became more likely to engage in symbolic exclusion, less educated persons became relatively less likely to do the same.

\section{Quantities of Interest from Preferred Model}

We use the preferred specification, shown as model 5 in Table 3, to extract some quantities of interest useful for gaining insight into the pattern of results. First we use the ZIP estimates to generate the probability of being a "structural zero" or, in our terms, a "true" categorical tolerant, by survey year, partitioned by cohort and according to black versus nonblack ethnoracial status (predicted probabilities of a successful outcome in the logit component of the equation). Figure 3 shows the results. We can see that the probability of being a categorical tolerant is higher in 2012 for members of all cohorts. However, the survey wave effect is much stronger for the youngest cohort and weakest for persons born between 1936 and 1954 .

As expected, the number of categorical tolerants who are not black is below the sample mean for persons reporting no dislikes in each survey (dashed lines), except for persons born after 1968 (in which case these two quantities are the same). Among blacks, on the other hand, the proportion of categorical tolerants is about the 

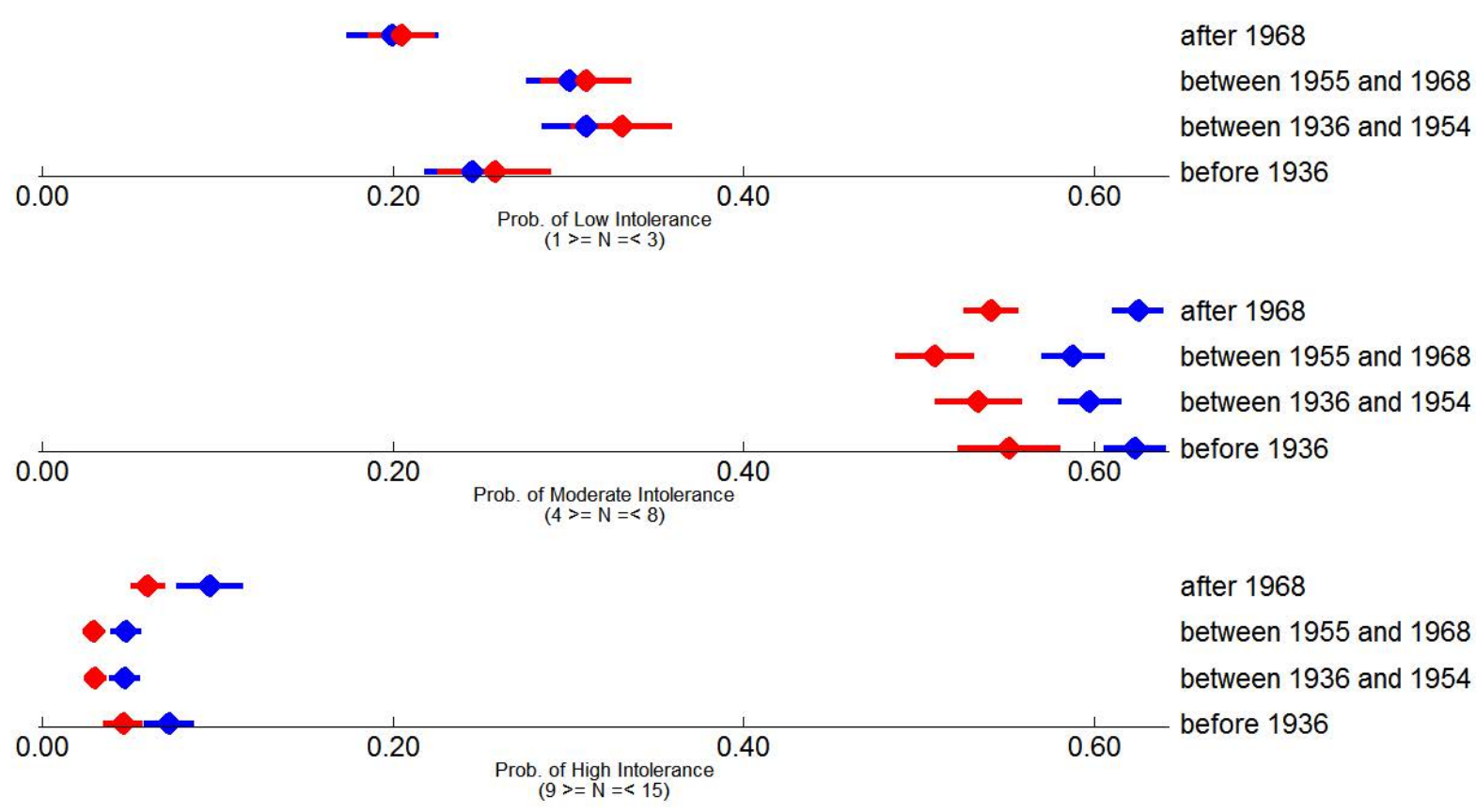

\section{2}

Figure 4: Predicted probability of falling at different levels of symbolic exclusion intensity from count component of the preferred zero-inflated Poisson specification by cohort group

same as the proportion of the sample in each survey reporting no dislikes, except for blacks born after 1955, among whom the number of categorical tolerants exceeds that of persons reporting no dislikes in the survey as a whole.

To examine quantities of interest from the Poisson (count) component of the equation, we divided the count portion outcome into three intervals ordered by increasing intolerance levels (number of dislikes): low (one to three); moderate (four to eight); and high (nine or more). Recall that this is the probability of expressing some number of dislikes for persons who are not categorically tolerant (e.g., have a non-zero risk of expressing at least one dislike). The analytic goal is to ascertain the extent to which cohort membership predicted membership in these three categories.

Figure 4 shows the results. We can see that for the most part there is very little cohort-based variation across surveys at either the low or high end of intolerance, with the probability of falling into each of these categories being essentially the same for all cohorts in 1993 and 2012. The exception is members of the most recently born (post-1968) cohort, who display a statistically discernable decline in the probability of high intolerance in 2012 in comparison to 1993. However, the key finding here is that there has been cohort movement in the "moderate" intolerance category, especially among younger cohorts. For the most part, it appears that Americans at risk of demonstrating some form of symbolic exclusion are less likely to do so at moderate levels in 2012 than they were in 1993. 

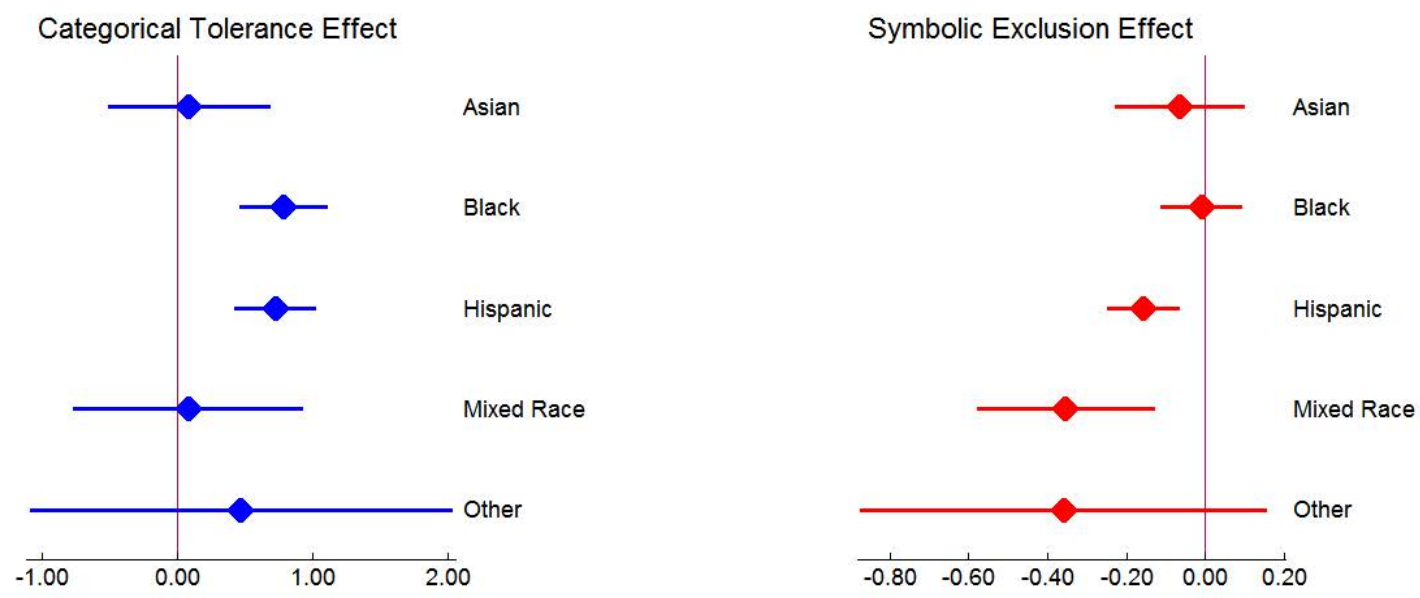

Figure 5: Regression plot (Jann 2014) of coefficient estimates for binary ethnoracial predictors for binary (blue marker, lefthand side) and count (red marker, righthand side) components of zero-inflated Poisson models predicting number of dislikes in 2012

\section{Categorical Tolerance and Ethnoracial Status in 2012}

It is clear that in addition to generation, ethno-racial status seems to be one of the most powerful predictors of the rise in categorical tolerance. Unfortunately, more detailed analysis of this factor's effect is hamstrung by the draconian (essentially binary) ethno-racial status coding in the merged GSS/SSI data. In this section we provide some additional insight into the role of race and ethnicity in the generation of categorical tolerance via a single-year cross-sectional analysis of the SSI 2012 survey, which includes a more detailed (and contemporary) set of ethno-racial identification labels.

Our strategy is to estimate a zero-inflated Poisson model predicting both categorical tolerance (in the binary component) and symbolic exclusion (in the count component) using a specification close to that in model 5 of Table 3, except that instead of binary predictors for "white" and "black" ethno-racial status, we include four binary predictors coding for self-identification as: (1) black/African American (12.27 percent); (2) Asian (4.53 percent); (3) Hispanic (16.05 percent); (4) mixed race (2.33 percent; $N=53$ ); and (5) other ( 0.48 percent; $N=11$ ). The implied comparison group in this model is self-identified whites. In this model the "survey year" predictor is naturally excluded, and the "cohort" predictors are replaced with a continuous input indexing the respondent's age, with quadratic and linear specifications of the age effect entered as predictors in the count and binary components of the model, respectively.

The coefficient estimates corresponding to the ethno-racial predictors for this model are plotted in Figure 5 (Jann 2014). In the figure, the diamond-shaped marker represents the coefficient estimate, and the horizontal line the 95 percent confidence interval based on the (robust) standard error estimate. For ease of interpretation, the zero (null) effect line is drawn on the vertical plane of the plot. On the left-hand side we show the logit estimates predicting membership in the categorical tolerant 
group, while on the righthand side we show the coefficient estimates for the Poisson regression (predicting symbolic exclusion among those at risk).

From the figure, it is clear that both symbolic exclusion patterns and categorical tolerance are not homogeneous in relation to ethno-racial categories. However, the effects of ethno-racial status are not necessarily symmetric across these two substantive outcomes because, as we have seen in the case of generational predictors, a variable that positively predicts membership in the categorical tolerant group does not necessarily imply that the same covariate reduces the likelihood of engaging in symbolic exclusion among those at risk of expressing at least one dislike.

For instance, though self-identification as black or African American has a strong and statistically significant effect on the odds of being a categorical tolerant $(t=4.75)$, the same predictor has a negligible and statistically null effect on the number of dislikes $(|t|=0.16)$. This suggests that the ethno-racial effect of being black is only confined to excluding individuals from those who could engage in some form of symbolic exclusion. The effects of self-identification as mixed race are opposite those for the binary predictor for black ethno-racial status. The mixed race predictor has a null effect on the chances of belonging to the categorical tolerant group $(|t|=0.19)$ but has a strong negative effect on the likelihood of engaging in symbolic exclusion $(t=-3.05)$. This is consistent with the idea that those who either "cross" or "blur" boundaries in one domain (i.e., ethno-racial identification) are also less likely to draw strong symbolic boundaries in other domains, such as musical taste (Wimmer 2013; Wimmer and Lewis 2010).

The case of Hispanics, on the other hand, does indicate the existence of concordant (opposite) effects on both the odds of membership in the categorical tolerant group $(t=4.73)$ and the likelihood of engaging in symbolic exclusion $(t=-3.30)$, increasing the odds of the former and decreasing the intensity of the latter. Given the tendency of Hispanics to be overrepresented in the categorically tolerant group, the growth of this segment of the population in the United States for the last two decades should be partially responsible for the increase in the proportion of the population that belongs to this category that we observe. Note that the small minority of respondents who chose "other" as their ethnoracial identity behave a lot like mixed race respondents in terms of the magnitude and direction of the point estimate, although with such a small number of cases the precision of this estimate is naturally very low.

Finally, an important observation is that persons who chose "Asian" as their ethno-racial identification are indistinguishable from whites, both in terms of the likelihood of belonging in the categorical tolerant group $(|t| t=0.30)$ and the intensity of their symbolic exclusion behavior $(|t| t=0.75)$. This is in contrast to the results pertaining to the other minority ethno-racial label predictors, which have either positive effects on categorical tolerance, negative effects on symbolic exclusion, or, in the case of Hispanics, both. This Asian difference is consistent with the triracial order model in the literature on race and ethnic relations (Bonilla-Silva 2004). This model posits explicit and implicit behavioral, attitudinal, and judgmental affinities between whites and Asians (and other "honorary white" groups), which increasingly put them in conflict with those of racialized members of selfidentified black and Hispanic populations. While triracial order theory has been 
evaluated mostly in the realm of explicitly social and political attitudes, we can see that there is crossover applicability to the domain of taste in keeping with Bryson's (1996) original hypothesis of predictable symmetries between political and aesthetic judgments (see also Bourdieu 1984; Laurison 2015).

\section{Discussion}

\section{The Rise of Categorical Tolerance: Young, Brown, and Nonelite}

True categorical tolerants, persons who exclude themselves from engaging in symbolic exclusion, have tripled as a segment of the U.S. population in the last two decades. Categorical tolerance is a distinctive phenomenon given the generalized propensity, foundational to most contemporary understandings in the sociology of taste, of persons to use cultural dislikes as a resource for symbolic exclusion and identity construction (Bryson 1996, 1997). As such, the growth in the tendency to categorically refuse to dislike seems to indicate the beginning of a fundamental shift in the ways that individuals use (or refuse to use) culture for these purposes (Swidler 2001).

The tripling of the size of the categorical tolerant group seems to be due primarily to generational replacement, as members of recently born cohorts are more likely to be categorical tolerants, and this cohort growth pattern seems to be (for the most part) linear. ${ }^{10}$ While generational replacement processes are likely a powerful driver of this development, they are not the only factor at play. In fact, the sociodemographic "seeds" of categorical tolerance seemed to have already been evident in 1993. This is given, for instance, by the fact that blacks were overrepresented by a factor of close to two to one in this group, and continue to be in 2012 by a comparable margin. This is a finding that to our knowledge had never been highlighted in the sociology of taste, but which is of considerable theoretical and substantive interest. ${ }^{11}$ Though relatively little has been written on the subject, it is clear that ethnoracial minorities in the United States do not use cultural dislikes in the same way that members of the white majority do (Carter 2006).

As noted at the outset, the pattern of "conspicuous openness to diversity" (Ollivier 2004) has been conceptualized as primarily characteristic of (presumably primarily Anglo-European) global, professional, educated elites (Saito 2011). Yet cultural tolerance among other groups, especially nonwhite and nonelite populations, continues to be a relatively underresearched topic (but see Warikoo 2007). Categorical tolerance in the United States seems to be one such overlooked pattern, displaying both an overrepresentation of nonwhites and an underrepresentation of traditional elites (i.e., the highly educated), in addition to the young. It is clear that by Bryson's standards, African Americans in the United States have been and continue to be "multicultural capitalists," but their approach is more likely to take the form of a refusal to engage in rejection of other cultures, manifested as categorical tolerance. The continuing expansion of this tendency and its possible acceleration in the wake of the continuing growth of the Hispanic population (who seem to follow the native black pattern in this respect) portend the rise of a new form of taste expression, one that eliminates dislikes from the judgmental tool kit of taste 
(at least in social surveys). This elimination in its turn may be driven by subjective and social motivations quite orthogonal to the conspicuous display of openness and acceptance of diversity of white global cosmopolitans in world culture (Boli and Lechner 2005; Saito 2011).

\section{The End of Symbolic Exclusion?}

Does the large increase in categorical tolerance signal the end of symbolic exclusion? Taking into consideration the entirety of the evidence considered above, the answer is a resounding no. First, outside of the growth of categorical tolerance, it is clear that once we consider those at risk of engaging in some form of symbolic exclusion, there has been a surprising steadiness or even an uptick in the tendency to engage in symbolic exclusion among the most privileged strata of the population. This includes both those privileged in the ethnoracial hierarchy (whites) and those privileged in the status and class hierarchy (college-educated respondents). Of particular importance is our failure to replicate Bryson's patterned tolerance effect in the 2012 data. This could be due to a true shift towards more symbolic exclusion in the taste judgments of the more educated, but alternatively this failure could be due to a substantively significant method effect. Because the SSI is self-administered online and the GSS is a live, in-home interview, it is possible that highly educated respondents were more likely to reveal their dislikes for certain genres in the self-administered context. Future research can investigate the possibility that highly educated respondents display more cultural tolerance in research settings that induce more concerns with self-presentation.

Generational mechanisms also seem unlikely to bring the end of symbolic exclusion any time soon. First, members of young cohorts (along with members of the oldest) are the most likely to engage in the practice in the first place. Second, there has only been a marginal decline, on average, in the tendency to engage in symbolic exclusion among members of younger cohorts. Detailed analyses, such as that shown in Figure 3, show generational stability in symbolic exclusion behavior at both extremes of the scale, and a period effect negatively affecting all cohorts for moderate levels. While members of the oldest cohort do appear to have become more tolerant over time, this is likely due to differential mortality and not to a shift in the minds and tastes of persons over time towards more cultural tolerance. One factor that does seem to bode well for a (moderate) decline in symbolic exclusion behavior is the rise of Hispanics as the largest minority group in the United States. As we saw in the cross-sectional analysis of the 2012 data, Hispanics (and multiracials) tend to engage in less symbolic exclusion behavior than blacks, Asians, and whites. This may be due to the fact that practical dispositions towards boundary crossing by members of recently arrived minority groups (e.g., bilingualism and biculturalism) lend themselves naturally to boundary blurring and crossing in the cultural realm ("multicultural capital").

\section{Directions for Future Research}

Our analysis, relying primarily on population-level survey data, raises as many questions as it answers. While survey data are appropriate for answering questions 
of representation, rates, overall likelihood, and the existence (or nonexistence) of broad phenomena (e.g., symbolic exclusion, patterned and categorical tolerance), it is relatively limited when it comes to shedding light on the relevant processes and mechanisms underlying the regularities so uncovered (Spillman 2014; Small 2009; Fishman and Lizardo 2013; Lizardo and Skiles 2012; Warde, Wright, and GayoCal 2008; Savage and Gayo 2011). As such we recommend that future research on the categorical tolerance phenomenon take a mixed-methods approach. In particular, given the demographic profile of categorical tolerants uncovered in this study (young, nonwhite, and nonelite), following a logic of theoretical (rather than random) sampling and using interview-based and other close-to-the-ground observational methods, useful for separating "sayings from doings" (Jerolmack and Khan 2014), might be useful in answering outstanding questions regarding the possibly distinct ways in which persons refuse to use culture for their collective identity-construction projects, or even whether they do in fact use culture for such purposes.

In this respect, it would be important to establish the nature and bounds of the explicit discourses and motivations in terms of the taste-expression and cultureconsumption habits of categorical tolerants. A variety of questions emerge in this respect. Does categorical tolerance in one domain translate to tolerance in other domains (both cultural and social)? How do categorical tolerants understand and justify their refusal to dislike? To what extent do young, nonwhite, nonelite tolerants echo the institutionalized discourse of conspicuous openness to diversity characteristic of global cosmopolitan elites? To what extent have they developed a discourse on openness that reflects their own concerns and institutional location? Is categorical tolerance as "conspicuous" for this group as it is for global elites? Finally, it would also be important to ascertain whether categorical tolerance exists across comparative national settings (Katz-Gerro 2002). Given the generalized findings regarding other phenomena, such as "omnivorousness," in the comparative literature (Peterson 2005; Lizardo and Skiles 2009; Purhonen and Wright 2013; Fishman and Lizardo 2013), it is unlikely that categorical tolerance is a phenomenon confined to the United States. However, there may exist important cross-national differences in the extent, intensity, socio-demographic composition, and generational timing of categorical tolerants as a group and categorical tolerance as an aesthetic orientation.

\section{Notes}

1 The main difference is that GSS uses a (stratified) random sampling design and is an in-person interview The SSI data were collected using a series of demographic filters to approximate a random sample, and using web-based administration. The sample was constructed with an oversample of college graduates in order to meet the methodological requirements of a different project. As a result, the sample falls short of true representation of the U.S. population in several ways. First, only one-quarter of respondents completed their education before taking any college courses, compared with 44 percent in the U.S. adult population, and 40 percent of the sample completed a bachelor's or graduate degree, compared with 28 percent in the population (U.S. Bureau of the Census 2012a). A second consequence of this college oversample is slight age skew, with a modest underrepresentation of 18-19-year-olds (who compose only 2.6 percent of the 
sample, compared to 3.9 percent of the U.S. adult population, U.S. Bureau of the Census $2012 \mathrm{~b}$ ), and an overrepresentation of men aged 70 and older (who are 8.4 percent of the sample but 4.9 percent of the U.S. adult population, U.S. Bureau of the Census 2012b).

2 Unfortunately, as of 1993, the GSS still used a simple "triracial" system to code ethnoracial status (white, black, other). This means that for all cross-survey comparisons we collapsed the SSI racial identification variable (which includes Asian, Hispanic, multiracial, and other as options) into the GSS 1993 categories. Hence the comparisons between blacks and "nonblacks" in what follows.

3 An important difference is that GSS 1993 uses an interviewer coding method to determine ethnoracial status, but in SSI 2012 this predictor is given via the respondent's self-identification. We reconciled the two codings under the assumption that, given the high correlation between self- and other-identification of ethnoracial status (especially on binary black/white determination), this introduces negligible error into the race predictor.

4 We use the Mann-Whitney (1947) test (command ranksum in Stata 12.1).

5 A Mann-Whitney test of the distribution of dislikes that excludes the zero and one categories returns clear evidence that this truncated distribution comes from the same population $(|\mathrm{z}|=0.46 ; \mathrm{p}=0.65)$.

6 As we will see below the education differences in the 2012 SSI survey are themselves misleading, since they are a byproduct of the fact that the group of respondents expressing zero dislikes is a mixture of those who are truly "categorical" in their behavior (those at no risk of ever expressing a dislike) and those who could have disliked a genre but did not; it is really among this last group that differences in the probability of expressing dislikes by education have dissipated over time.

7 Note that the "increasing" tolerance of persons in the oldest surviving cohorts will likely generate a (spurious) "cohort effect" that will show up as increasing "intolerance" among younger members.

8 This is the "zero-inflated" Poisson model with robust standard errors (zip with option vce(robust) in Stata 12.1). This is a two-stage model that consists of a first-stage logit regression modelling the probability of being a "structural zero" (essentially separating those respondents who would not engage in symbolic exclusion under any circumstances from those who could engage in it), and then modelling the counts among this last set of respondents using a Poisson regression model. While described sequentially (two stages) for convenience, parameter estimates for both the logit and Poisson models are estimated simultaneously via maximum-likelihood (see Long and Freese 2005:396-8).

9 In a maximum likelihood estimation framework, this is given by the Vuong statistic (Long and Freese 2005:408-409) comparing a mixed binary-count model against its (nested) count-only counterpart. In Stata 12.1 this is done by specifying the zip command with the vuong option.

10 Nonlinearity in the categorical tolerance-cohort relationship is introduced by the greater propensity of those born before 1936 to be categorical tolerants. Beginning with the post-1936 cohort, representation in the categorical tolerant group increases as birth year increases.

11 This is possible because the dislikes outcome in GSS 1993 was originally analyzed using the linear regression method (Bryson 1996) and has been subsequently re-analyzed using latent class analysis (Tampubolon 2008) or Relational Class Analysis (Goldberg 2011), but has never been treated as a proper count outcome. 


\section{References}

Boli, John and Frank Lechner. 2005. World Culture: Origins and Consequences. Malden, MA: Blackwell.

Bonilla-Silva, Eduardo. 2004. "From Bi-racial to Tri-racial: Towards a New System of Racial Stratification in the USA." Ethnic and Racial Studies 27:931-950. http://dx . doi .org/10. 1080/0141987042000268530.

Bourdieu, Pierre and Jean Claude Passeron. 1977. Reproduction in Education, Society, and Culture. London: Sage.

Bourdieu, Pierre. 1984. Distinction: A Social Critique of the Judgment of Taste. Cambridge, MA: Harvard University Press.

Bryson, Bethany. 1996. "Anything but Heavy Metal: Symbolic Exclusion and Musical Dislikes." American Sociological Review 61(5):884-899. http://dx.doi.org/10.2307/ 2096459.

Bryson. 1997. "What About the Univores? Musical Dislikes and Group-based Identity Construction among Americans with Low Levels of Education." Poetics 25:141-156.

Carter, Prudence L. 2006. "Straddling boundaries: Identity, culture, and school." Sociology of Education 4:304-328. http://dx.doi.org/10.1177/003804070607900402.

Collins, Randall. 1975. Conflict Sociology: Toward an Explanatory Science. New York: Academic Press.

Davis, James Allan, Tom W. Smith, and Peter V. Marsden. 2007. General Social Surveys, 1972-2006. NORC ed. Chicago: National Opinion Research Center (producer); Storrs, CT: The Roper Center for Public Opinion Research, University of Connecticut (distributor).

DiMaggio, Paul. 1987. "Classification in Art." American Sociological Review 52:440-455. http://dx.doi.org/10.2307/2095290.

DiMaggio, Paul. 1996. "Are Art-Museum Visitors Different from Other People? The Relationship between Attendance and Social and Political Attitudes in the U.S." Poetics 24:161-180. http://dx.doi.org/10.1016/S0304-422X (96) 00008-3.

Fishman, Robert M. and Omar Lizardo. 2013. "How Macro-historical Change Shapes Cultural Taste: Legacies of Democratization in Spain and Portugal." American Sociological Review 78:213-23. http://dx.doi.org/10.1177/0003122413478816.

Frank David John and John W. Meyer. 2002. "The Profusion of Individual Roles and Identities in the Postwar Period." Sociological Theory 20:86-105. http://dx.doi.org/10. 1111/1467-9558.00152.

Goldberg, Amir. 2011. “Mapping Shared Understandings Using Relational Class Analysis: The Case of the Cultural Omnivore Reexamined." American Journal of Sociology 116(5):1397-1436. http://dx.doi.org/10.1086/657976.

Holt, Douglas. 1997. "Distinction in America? Recovering Bourdieu's Theory of Taste from its Critics." Poetics 25:93-120. http://dx. doi .org/10.1016/S0304-422X (97) 00010-7.

Hout, Michael and Claude S. Fisher. 2002. "Why More Americans Have No Religious Preference: Politics and Generations." American Sociological Review 67:165-190. http: //dx.doi.org/10.2307/3088891.

Jann, Ben. 2014. "Plotting regression coefficients and other estimates." Stata Journal 14(4):708737.

Jerolmack, Colin and Shamus Khan. 2014. "Talk is Cheap: Ethnography and the Attitudinal Fallacy." Sociological Methods and Research 43(2):178-209. http://dx . doi .org/10.1177/ 0049124114523396. 
Katz-Gerro, Tally. 2002. "Highbrow cultural consumption and class distinction in Italy, Israel, West Germany, Sweden, and the United States." Social Forces 81:207-229. http: //dx.doi.org/10.1177/0049124114523396.

Lamont, Michèle and Annette Lareau. 1988. "Cultural Capital: Allusions, Gaps and Glissandos in Recent Theoretical Developments." Sociological Theory 6:153-168. http: //dx.doi.org/10.2307/202113.

Lamont, Michèle and Virág Molnár. 2002. "The Study of Boundaries in the Social Sciences." Annual Review of Sociology 28:167-195. http://dx.doi .org/10.1146/annurev . soc . 28. 110601.141107.

Laurison, Daniel. 2015. “The Willingness to State an Opinion: Inequality, Don't Know Response, and Political Participation." Sociological Forum (forthcoming).

Lizardo, Omar. 2005. "Can Cultural Capital Theory be Reconsidered in the Light of World Policy Institutionalism? Evidence from Spain." Poetics 33:81-110. http://dx. doi .org/ $10.1016 / j$. poetic. 2005.02 .001 .

Lizardo, Omar. 2006. “How Cultural Tastes Shape Personal Networks." American Sociological Review 71:778-807. http://dx.doi.org/10.1177/000312240607100504.

Lizardo, Omar. 2008. "The Question of Culture Consumption and Stratification Revisited." Sociologica 2/2008. http://dx.doi.org/10.2383/27709..

Lizardo, Omar and Sara Skiles. 2009. "Highbrow Omnivorousness on the Small Screen? Cultural Industry Systems and Patterns of Cultural Choice in Europe." Poetics 37:1-23. http://dx.doi.org/10.1016/j.poetic.2008.10.001.

Lizardo, Omar and Sara Skiles. 2012. "Reconceptualizing and Theorizing 'Omnivorousness': Genetic and Relational Mechanisms." Sociological Theory 30:260-280. http://dx.doi. org/10.1177/0735275112466999.

Long, J. Scott and Jeremy Freese. 2005. Regression Models for Categorical Dependent Variables Using Stata. 2nd ed. College Station, TX: Stata Press.

Mahon, Maureen. 2000. "Black Like This: Race, Generation, and Rock in the Post-Civil Rights Era." American Ethnologist 27:283-311. http://dx.doi.org/10.1525/ae.2000. 27.2 .283$.

McPherson, Miller, Lynn Smith-Lovin, and Matthew E. Brashears. 2006. "Social Isolation in America: Changes in Core Discussion Networks Over Two Decades." American Sociological Review 71:353-375. http://dx.doi .org/10.1177/000312240607100301.

Ollivier, Michèle. 2004. “Towards a Structural Theory of Status Inequality: Structures and Rents in Popular Music and Tastes." Research in Social Stratification and Mobility 21:187-213. http://dx.doi.org/10.1016/S0276-5624(04)21010-1.

Ollivier, Michèle. 2008. "Modes of openness to cultural diversity: Humanist, populist, practical, and indifferent." Poetics 36:120-147. http://dx.doi .org/10.1016/j . poetic. 2008.02 .005$.

Peterson, Richard A. 2005. "Problems in Comparative Research: The Example of Omnivorousness." Poetics 33:257-282. http://dx.doi .org/10.1016/j .poetic .2005 .10.002.

Peterson, Richard A. and Albert Simkus. 1992. “How Musical Tastes Mark Occupational Status Groups." Pp. 152-186 in Cultivating Differences: Symbolic Boundaries and the Making of Inequality, edited by Michele Lamont and Marcel Fournier. Chicago: University of Chicago Press.

Prieur, Annick and Mike Savage. 2011. “Updating Cultural Capital Theory: A Discussion Based on Studies in Denmark and in Britain." Poetics 39:566-580. http://dx.doi .org/ $10.1016 / j$. poetic. 2011.09 .002 . 
Purhonen, Semi and David Wright. 2013. "Methodological Issues in National-Comparative Research on Cultural Tastes: The Case of Cultural Capital in the UK and Finland." Cultural Sociology 7:257-273. http://dx.doi .org/10.1177/1749975512473462.

Saito, Kiro. 2011. "An Actor-Network Theory of Cosmopolitanism." Sociological Theory 29:124-149. http://dx.doi.org/10.1111/j.1467-9558.2011.01390.x.

Savage, Mike and Modesto Gayo. 2011. "Unravelling the Omnivore: A Field Analysis of Contemporary Musical Taste in the United Kingdom." Poetics 39:337-357. http: //dx.doi.org/10.1016/j.poetic.2011.07.001.

Small, Mario Luis. 2009. "'How Many Cases Do I Need’: On Science and the Logic of Case Selection in Field-Based Research?" Ethnography 10:5-38. http://dx.doi .org/10.1177/ 1466138108099586.

Spillman, Lyn. 2014. “Mixed Methods and the Logic of Qualitative Inference." Qualitative Sociology 37:189-205. http://dx . doi .org/10.1007/s11133-014-9273-0.

Stouffer, Samuel C. 1955. Communism, Conformity, and Civil Liberties. New York: Doubleday. Swidler, Ann. 2001. Talk of Love: How Culture Matters. Chicago: University of Chicago Press.

Tampubolon, Gindo. 2008. "Revisiting omnivores in America circa 1990s: The exclusiveness of omnivores?" Poetics 36:243-264. http://dx.doi.org/10.1016/j.poetic.2008.02. 007.

U.S. Census Bureau. 2012a. “Table 6. Resident Population by Sex, Race, and Hispanic-Origin Status: 2000 to 2009." Statistical Abstract of the United States: 2012. http: / /www . census . gov/prod/2011pubs/12statab/pop.pdf.

U.S. Census Bureau. 2012b. “Table 7. Resident Population by Sex and Age: 1980 to 2010." Statistical Abstract of the United States: 2012. http://www . census .gov/prod/2011pubs/ 12 statab/pop.pdf.

van Eijck, Koen. 2001. "Social Differentiation in Musical Taste Patterns." Social Forces 79:1163-1185. http://dx.doi.org/10.1353/sof .2001.0017.

Warde, Alan, David Wright, and Modesto Gayo-Cal. 2008. "The omnivorous orientation in the UK." Poetics 36:148-165. http://dx.doi.org/10.1016/j .poetic. 2008.02.004.

Warikoo, Natasha. 2007. "Racial Authenticity among Second Generation Youth in Multiethnic New York and London." Poetics 35:388-408. http://dx.doi.org/10.1016/j.poetic. 2007.09 .001$.

Wimmer, Andreas. 2013. Ethnic Boundary Making: Institutions, Power, Networks. Oxford: Oxford University Press. http://dx.doi.org/10.1093/acprof : oso/9780199927371. 001.0001.

Wimmer, Andreas and Kevin Lewis. 2010. "Beyond and Below Racial Homophily: ERG Models of a Friendship Network Documented on Facebook." American Journal of Sociology 116:583-642. http://dx.doi.org/10.1086/653658.

Acknowledgements: This research was made possible by funding from NSF Doctoral Dissertation Improvement Grant (Award ID SES-1203426) awarded to the second author (with the first author as PI) and a University of Notre Dame Institute for Scholarship in Liberal Arts Graduate Student Research Award awarded to the second author.

Omar Lizardo: Department of Sociology, University of Notre Dame. E-mail: olizardo@nd.edu.

Sara Skiles: Department of Sociology, University of Notre Dame. E-mail: sskiles@nd.edu. 